\title{
Search for Electric Dipole Moments of Charged Particles with Polarized Beams in Storage Rings
}

\author{
Paolo Lenisa*† \\ Universitá di Ferrara and INFN, Italy \\ E-mail: lenisa@fe.infn.it
}

The observation of a non zero electric dipole moment of a fundamental particle would represent a clear sign of New Physics beyond the Standard Model. An experimental program is presently pursued by the JEDI Collaboration at the COSY storage ring of the Forschungszentrum Jülich (Germany) to provide the first ever measurement of the electric dipole moment of the deuteron. The gained experienced and developed technologies result important in perspective of the design and realization of a future dedicated storage ring.

The 18th International Workshop on Polarized Sources, Targets, and Polarimetry, PSTP2019 23-27 September, 2019

Knoxville, Tennessee

* Speaker.

${ }^{\dagger}$ On behalf of the JEDI Collaboration 


\section{Introduction}

One of the most intriguing questions in cosmology and perhaps in all of physics is: âĂIJWhy is there so much matter in the Universe and so little antimatter?âĂİ. Until today, there is no evidence for any primordial antimatter within our galaxy or even beyond. There is no indication for any form of co-existence of matter and antimatter in clusters or galaxies within our Universe. Hence, it is usually concluded that our visible Universe is made entirely of matter and is intrinsically matter non-symmetric. According to the combined Standard Models of cosmology and particle physics it is expected that at the end of the inflationary epoch - following the Big Bang - the number of particles and antiparticles were in extreme balance, yet somehow the laws of physics contrived to act differently on matter and antimatter in order to generate the current imbalance. Interestingly, one of the necessary physics mechanisms required for such effects âĂŞ namely CP-violation âĂŞ is very small in the Standard Model (SM) of particle physics and thus is only able to account for a tiny fraction of the actual asymmetry.

While particle physics at accelerators celebrated its latest success with the discovery of the Higgs boson, culminating in a series of discoveries all consistent with the SM, the chances have grown up in recent years that new physics could be at mass scales beyond the reach of current or future collider experiments. This prospect, in combination with astrophysical observations (e.g. dark matter, neutrino oscillations), not explained by the SM has stimulated interest in high-precision physics. One such search for new physics is the quest for electric dipole moments (EDMs) in fundamental particles.

An EDM originates from a permanent electric charge separation inside the particle. In its centre-of-mass frame, the ground state of a subatomic particle has no direction at its disposal except its spin, which is an axial vector, while the charge separation (EDM) corresponds to a polar vector. If such a particle possesses an EDM, it must violate both parity $(\mathrm{P})$ and time-reversal $(\mathrm{T})$ invariance (Fig. 1).

If the combined CPT symmetry is to be valid, T violation also implies breaking of the combined CP symmetry. The Standard Model predicts the existence of EDMs, but their sizes fall many orders of magnitude below the sensitivity of current measurements and still far below the expected levels of projected experiments. An EDM observation at a much higher value might be interpreted as a sign of new physics beyond the current Standard Model (BSM).

Researchers have been searching for EDMs of neutral particles, especially neutrons, for more than 60 years, but, despite a constant increase in sensitivity, the experiments have come up only with upper bounds, nevertheless providing useful constraints on BSM theories (Fig. 2).

\section{EDM search in storage rings}

More recently, a new class of experiments based on storage rings has been proposed to improve the sensitivity of the measurements and eventually be able to measure the EDM of charged particles (such as the proton, deuteron or helion). The measuring principle is straightforward: a radial electric field is applied to an ensemble of particles circulating in a storage ring with their polarization vector (or spin) initially aligned with their momentum direction. The existence of an 


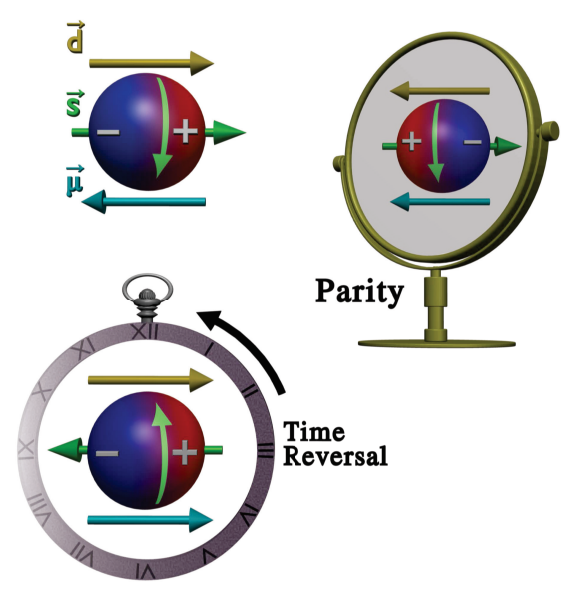

Figure 1: Naïve representation of a fundamental particle as a spherical object with an asymmetric charge density (upper left). The particle mirror image is represented on the right, and its time-reversal at the bottom. The particle spin defines (s) a direction in space. Both $\mathrm{P}$ and $\mathrm{T}$ transformations leave the magnetic dipole moment $(\mu)$ antiparallel to the spin while change the relative orientation of the electric dipole moment $(\mathbf{d})$. Therefore, the original particle can be distinguished from its mirror or time reversal image.

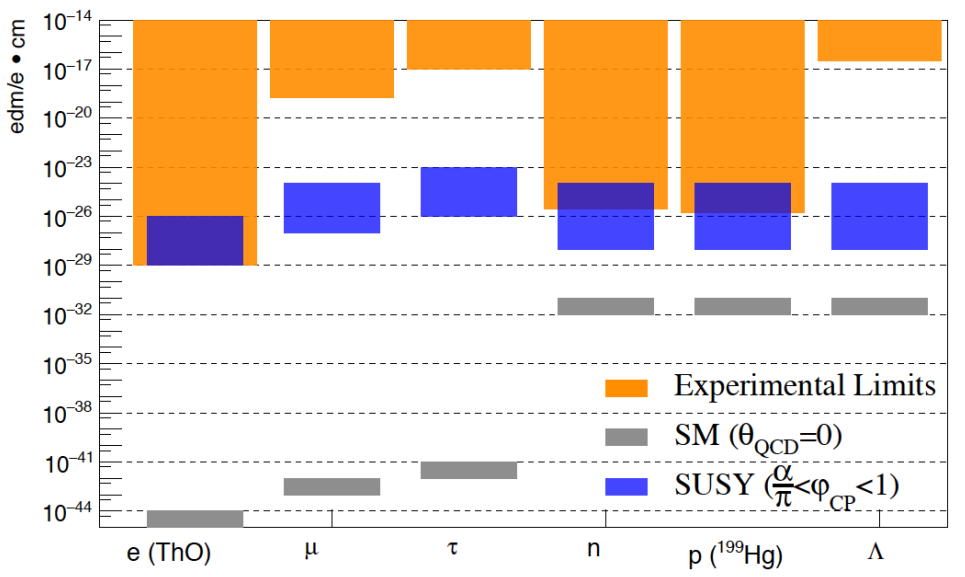

Figure 2: Experimental upper limits for the EDMs of different particles (red bars) plotted together with the prediction from SUSY (blue bands) and the Standard Model (grey bands). No experimental limit for the deuteron exists yet.

EDM would generate a torque that slowly rotates the spin out of the plane of the storage ring and into the vertical plane (Fig. 3).

This slow change in the vertical polarization is measured by sampling the beam with elastic scattering off a carbon target and looking for a slowly increasing left-right asymmetry in the scattered particle flux. For an EDM of $10^{-29} \mathrm{e} \cdot \mathrm{cm}$ and an electric field of $10 \mathrm{MV} / \mathrm{m}$, this would happen at an angular velocity of $3 \times 10^{-9} \mathrm{rad} / \mathrm{s}$ (about $1 / 100$ of degree per day!). This requires the measurement to be sensitive at a level never reached before in a storage ring.

These requirements imply that for a statistically significant result, the polarization in the ring plane must be kept for times on the order of a thousand seconds during a single fill of the ring 


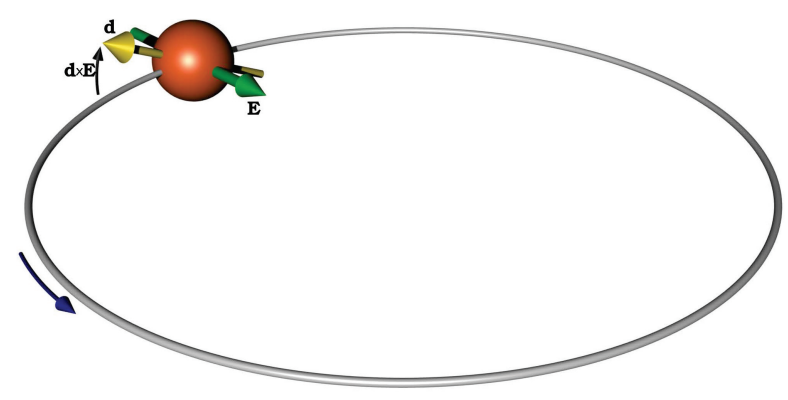

Figure 3: Measuring principle of a charged particle EDM in a storage ring. A radial electric field is applied to an ensemble of particles circulating in a storage ring with polarization vector aligned to their momentum: the existence of an EDM, would generate a torque that slowly rotates the spin out of the ring plane into the vertical direction.

and the scattering asymmetry from carbon must reach levels above $10^{-6}$ in order to be measurable within a year of running.

\section{Achievements at the COSY storage ring}

At the Cooler Synchrotron COSY located at the Forschungszentrum-Jülich (FZJ) (Fig. 4), the JEDI Collaboration [1] is working on a series of feasibility studies for the EDM experiment in a to-be-built dedicated storage ring. The COSY ring, able to store both polarized proton and deuteron beams, is an ideal machine for the development and commissioning of the necessary technology.

\subsection{Measurement of the spin-tune}

Following the commissioning of a measurement system that stores the clock time of each recorded event in the beam polarimeter, some major achievements have been already realized. The polarized beam is injected into COSY with the polarization vertical. Operating a radio-frequency solenoid for a brief period turns the polarization into the ring plane and subsequently the measurements are started. Above all, it was possible to unfold for the first time the rapid rotation of the polarization in the ring plane $(\approx 120 \mathrm{kHz})$ arising from the gyromagnetic anomaly. The spin tune (i.e. the number of spin precessions per turn) has been measured with a precision better than $10^{-10}$ in a cycle of 10 seconds that possibly represents the most precise measurement ever performed in a storage ring (Fig. 5) [2].

\subsection{Phase-locking the spin precession}

In a machine with purely magnetic deflection and focusing like COSY, it is not possible to freeze the spins. Using an RF device that operates on a harmonic of the spin-precession frequency is the only possible approach toward an EDM measurement in COSY. In order to achieve a good precision for such a measurement, phase-locking is necessary, making sure that phase between the spin precession and the device RF is maintained throughout the measurement. To this end, a feedback system has been developed that stabilizes the phase of the spin precession relative to the phase of an RF devices, providing a so-called phase-lock. The feedback system maintains the resonance frequency, and the phase between spin precession and device RF (e.g., solenoid or Wien 


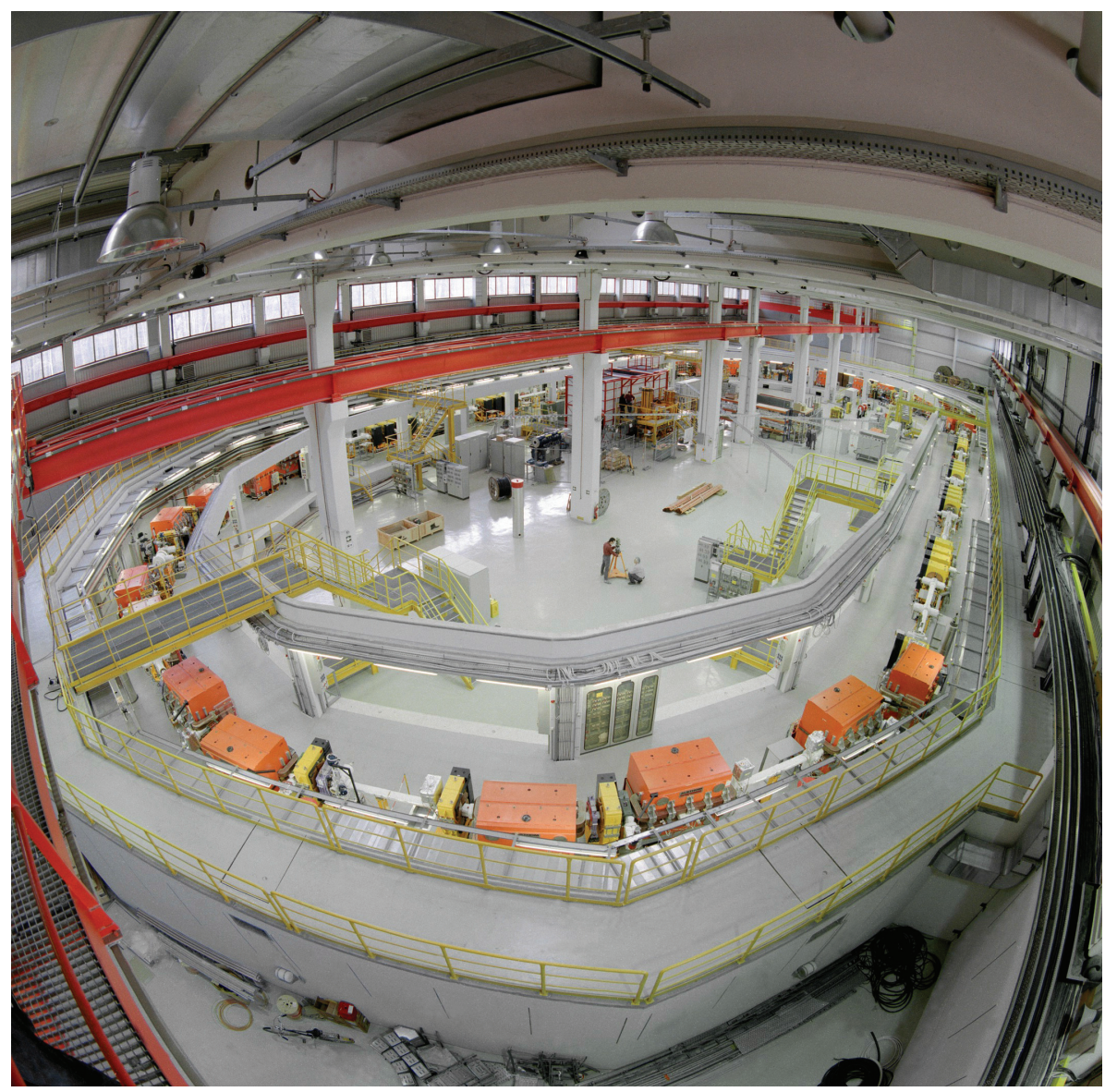

Figure 4: The COSY storage ring at the Forschungszentrum Jülich.

filter). As a major achievement, an error of the phase-lock of $\phi_{s}=0.21 \mathrm{rad}$ has been achieved. In the presence of a long spin-coherence time, phase-locking of the in-plane polarization can be viewed as providing a co-magnetometer for the resonant buildup of a vertical polarization component using an RF Wien filter (Fig. 6) [4])

\subsection{Spin-coherence time}

Another milestone was the achievement of polarization lifetimes in the ring plane longer than $1000 \mathrm{~s}$ (Fig. 7) [3].

Maintaining the polarization in the ring plane requires the cancellation of effects that may cause the particles in the beam to differ from one another. Bunching and electron cooling the beam serves to remove much of this spurious motion. However particle path lengths around the ring may differ if particles in the beam have transverse oscillations with different amplitudes. The effect of these differences on polarization decoherence may be removed by applying correcting sextupole fields to the ring. As a result, the polarization lifetimes now reach the required duration for the EDM experiment. 


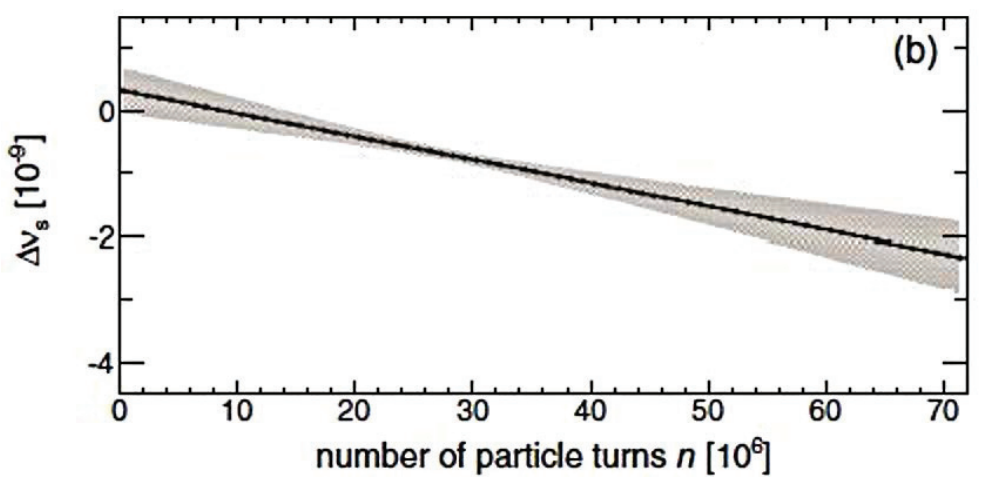

Figure 5: Deviation of the spin tune $v_{s}$ defined as the number of spin precessions per turn, as a function of the number of turns in the ring. At $\mathrm{t}=38 \mathrm{~s}$ (about $28 \times 10^{6}$ turns), the interpolated spin tune amounts to $(16097540628.3 \pm 9.7) \times 10^{11}$, which represents the most precise measurement of this quantity ever performed.

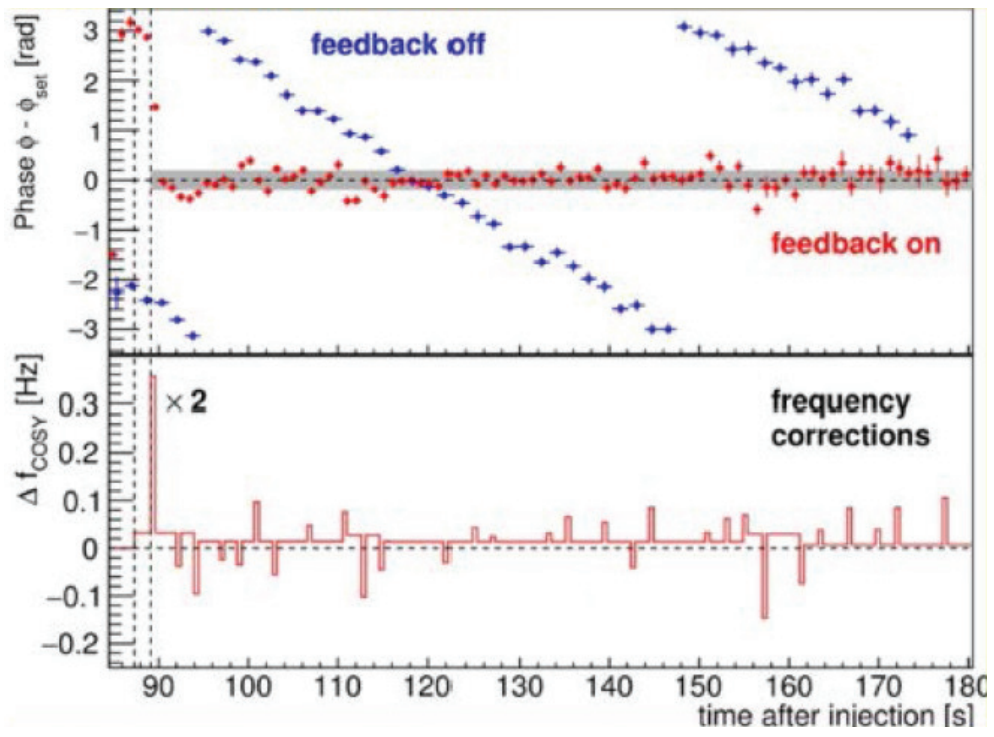

Figure 6: Operation of the active spin-feedback system. Spin-phase of the beam relatively to en external reference without and with use of the feedback system (upper picture) together with correction signals (lower picture).

\subsection{Spin-tune mapping}

JEDI developed a new method to investigate magnetic machine imperfections based on the highly accurate determination of the spin-tune. This spin-tune mapping technique used the two available cooler solenoids of COSY as (makeshift) spin rotators to generate artificial imperfection fields (Fig. 8, left panel). The measurement of the shifts of the spin tune as function of the spin kicks of the two solenoids yields the map [5]. The location of the saddle point of the map determines the tilt of the stable spin axis caused by the magnetic imperfections (Fig. 8, right panel). It is possible to control the background to the direction of the stable spin axis $\vec{c}$ from magnetic dipole moment rotations at a level $\Delta c=2.8 \times 10^{-6} \mathrm{rad}$ [5]. The systematics-limited sensitivity for a deuteron EDM measurement at COSY amounts to $\sigma_{d}=10^{-20} \mathrm{e} \cdot \mathrm{cm}$. 


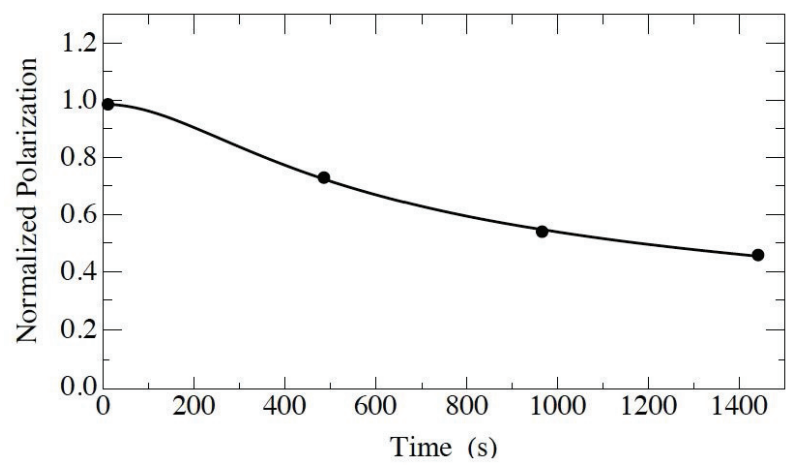

Figure 7: One of the longest polarization lifetimes recorded for the COSY ring. Measurements made at four separate times (to conserve beam) are matched to a depolarization curve that assumes a Gaussian distribution of transverse oscillation amplitudes. The half-life of the polarization is $1173 \pm 172 \mathrm{~s}$, which is three orders of magnitude longer than previous results using electron beams.

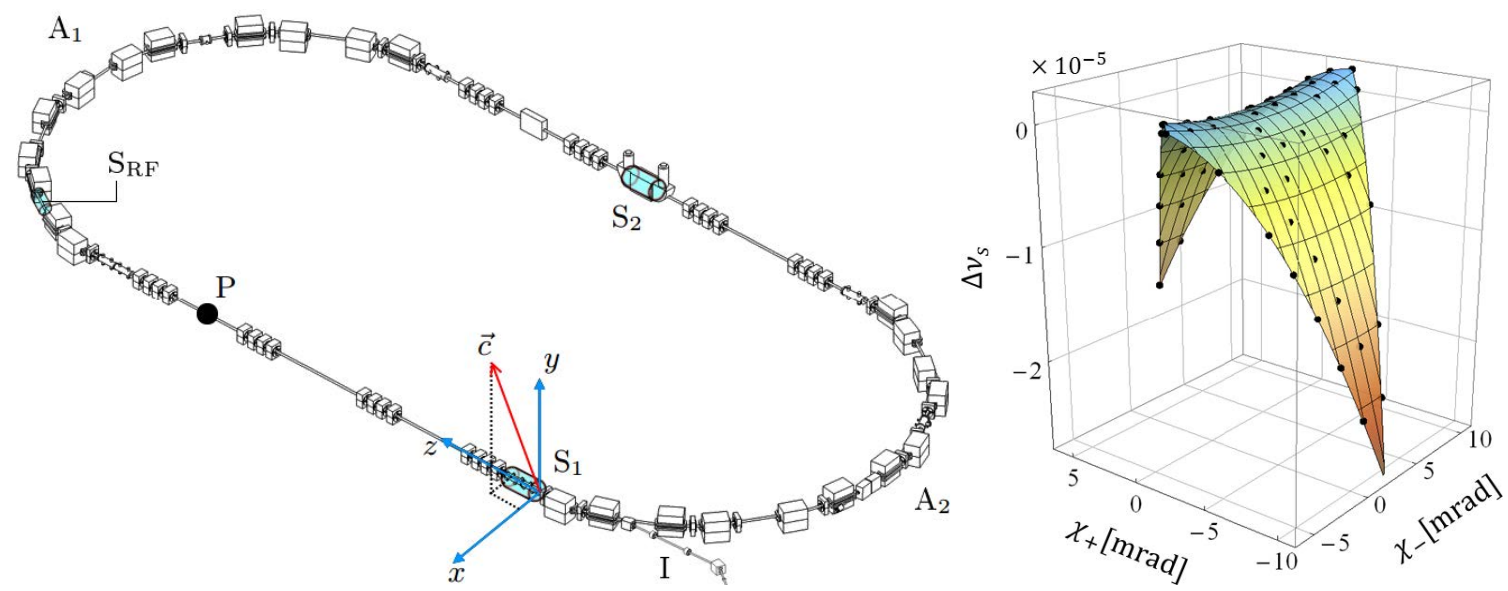

Figure 8: Left: the COSY storage ring with the solenoids imployed in the spin-tune mapping. Right: spin tune map. The saddle point determines the tilt of the stable spin axis caused by magnetic imperfections.

\section{Towards the first measurement of the deuteron EDM}

In 2016 the European Research Council awarded an Advanced Research Grant to the Juelich group, supporting further R-D efforts [8]. The goal of the project is to conduct the first ever measurement of the deuteron EDM. Since at COSY the polarization cannot be maintained parallel to its velocity, a novel device called a radiofrequency Wien filter [6] has been developed and installed in the ring to slowly accumulate the EDM signal: the filter influences the spin motion without acting on the particleâĂŹs orbit. The idea is to exploit the electric fields created in the particle rest system by the magnetic fields of the storage-ring dipoles (Fig. 9).

As the particle's spin precesses with a different frequency with respect to the velocity, the net contribution to the polarization buildup coming from the motional E-fields per turn would average to zero. The radiofrequency-Wien filter, synchronized with the spin precession frequency, restores the parallelism between spin and momentum and allow the polarization build-up to take place.

In the EDM experiment, the radiofrequency-Wien filter is rotated so that the B field points in 


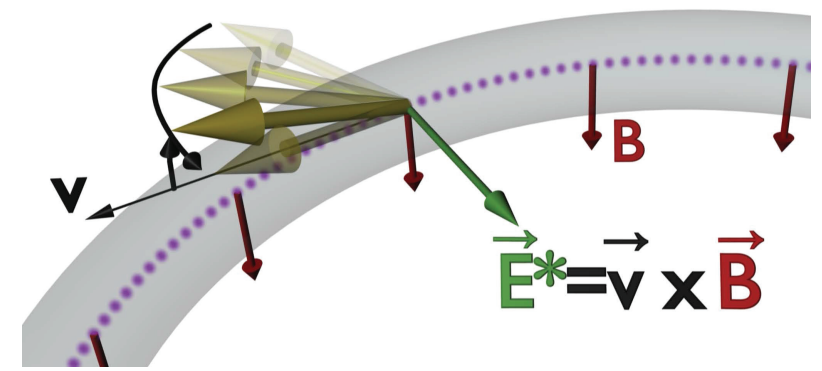

Figure 9: First measurement of the deuteron EDM as planned at COSY. The spin precesses in the vertical magnetic fi eld of the dipoles and feels a torque caused by the interaction of the EDM with the electric motional field. To allow for polarization buildup to occur, an RF-Wien filter will be used to control the relative phase between spin and momentum

the vertical direction and consequently acts on the spins of the particles precessing in the horizontal plane. To accomplish to the task, the frequency of the Wien filter is locked to the spin motion of the particles by a novel developed spin-feedback system [4]. The most significant challenges come from the management of systematic errors. Small imperfections in the placement and orientation of ring elements may cause stray field components that generate the accumulation of an EDM-like signal. The experiment is most sensitive to radial magnetic fields and vertical electric fields. Similar effects may arise through the non-commutativity of spurious rotations within the ring system. Efforts are underway to model these effects through spin tracking supported with beam testing. Eventually, many such effects may be reduced or eliminated by comparing the signal accumulation rates seen with beams traveling in opposite directions in the storage ring.

\subsection{Measurement of EDM-like polarization buildup}

EDM induced vertical polarization oscillations in an experimental situation with an RF- Wien filter can generally be described by:

$$
p_{y}(t)=\operatorname{asin}\left(\Omega_{p y} t+\phi_{R F}\right)
$$

The associated EDM resonance strength $\varepsilon_{E D M}$ can be defined as the ratio of angular frequency $\Omega_{p y}$ relative to the orbital angular frequency $\Omega_{r e v}$ in the machine:

$$
\varepsilon_{E D M}=\frac{\Omega_{p y}}{\Omega_{r e v}}
$$

The term EDMI applies to the case that only the EDM contributes to $\Omega_{p y}$. In practice, the resonance strength will receive contributions from other sources, such as rotations of the RF Wien filter and solenoidal fields in the ring that generate unwanted spin kicks.

$\varepsilon_{E D M}$ can be determined from the measured initial slopes $\left.\dot{p}_{y}\right|_{t=0}$ of the polarization buildup through a variation of the RF phase $\phi_{R F}$ using the phase-lock (see Fig. 10:

$$
\varepsilon_{E D M}=\frac{p_{y}(t) \mid t=0}{a \cos \phi_{R F}} \cdot \frac{1}{\Omega_{r e v}}
$$




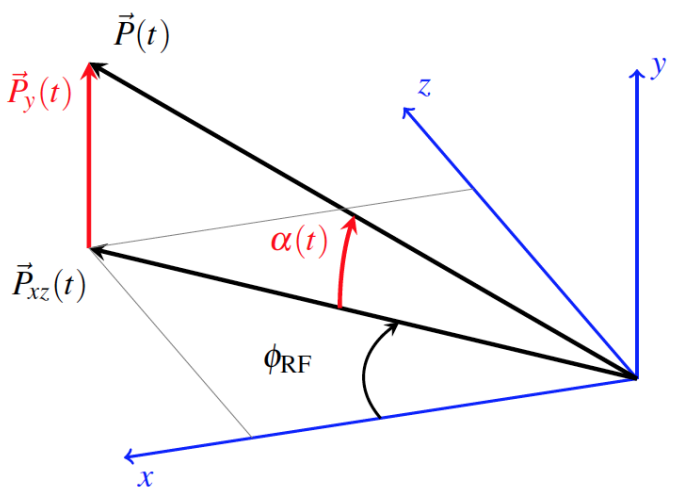

Figure 10: The deuteron spins are precessing in the horizontal (xz) plane and the RFWien filter is running on the corresponding frequency with a certain RF phase $\phi_{R F}$ that is maintained using the phase-locking system

The first measurements of EDM-like buildup signals by JEDI are shown in Fig. 11. Both plots show the rate of the out-of-plane rotation angle $\left.\dot{\alpha}\right|_{t=0}$ as function of the Wien filter RF phase $\left(\phi_{R F}\right)$ for different rotations of the RF Wien filter around the beam axis $\left(\phi_{r o t}^{W F}\right)$ and different spin rotations in the Snake solenoid $\left(\chi_{\text {rot }}^{\text {Snake }}\right)$. Variations of $\phi_{\text {rot }}^{W F}$ and $\chi_{\text {rot }}^{\text {Snake }}$ affect the pattern of observed initial slopes $\dot{\alpha}$. During the measurements the magnets of the electron cooler were switched off altogether on flattop to reduce unwanted spin precessions of the stored particles in the cooler magnets.

The fact that there is a buildup for the case with vertical B-field (red-curves) points to a nonvertical stable spin axis, possibly caused by misalignments in the ring. It roughly corresponds to an EDM at the $10^{-18} \mathrm{e} \cdot \mathrm{cm}$ level. To disentangle the real-EDM induced signal from the EDMlike signals coming from spurious magnetic field dedicated spin-tracking codes are required. They will be able simulate the EDM-like signal coming from the misalignments of the magnets and eventually provide the first experimental upper limit to the deuteron EDM.

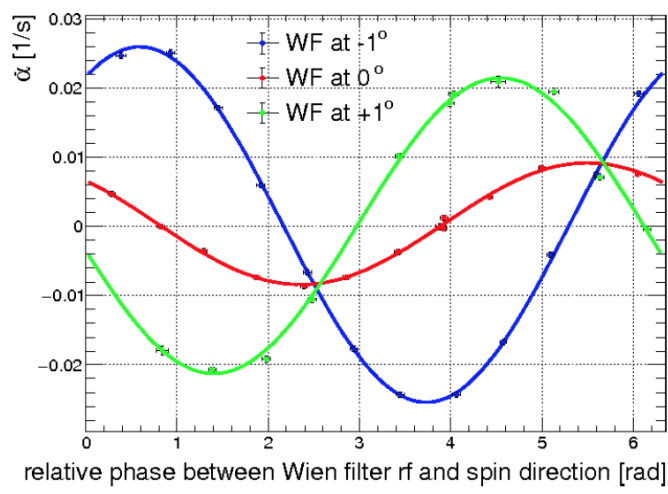

(a) $\dot{\alpha}$ for $\phi_{\mathrm{rot}}^{\mathrm{WF}}=-1^{\circ}, 0^{\circ},+1^{\circ}$ and $\chi_{\mathrm{rot}}^{\text {Snake }}=0$.

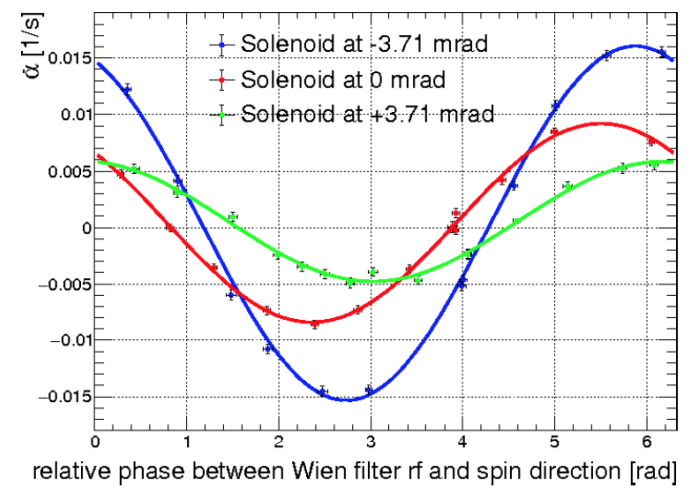

(b) $\dot{\alpha}$ for $\chi_{\text {rot }}^{\text {Snake }}=-1,0,+1^{\circ}$ and $\phi_{\text {rot }}^{\mathrm{WF}}=0$.

Figure 11: Rate of the out-of-plane rotation angle $\left.\dot{\alpha}\right|_{t=0}$ as function of the Wien filter RF phase $\phi_{R F}$ for two situations. In panel (a), only the RF Wien filter is rotated around the beam axis, and in (b) only the Siberian snake solenoid in the opposite straight section of COSY (see Fig. 1) rotates the spins around the beam axis. 


\section{Next steps}

\subsection{From JEDI to CPEDM: a prototype storage ring}

In view of the various technical challenges involved in building the final all-electric ring, as e.g., described in [8], as next step, the CPEDM collaboration decided to design and build a demonstrator ring for charged-particle EDM searches. The new CPEDM collaboration, which evolved out of the success and the achievements of JEDI, brings together scientists from CERN and the JEDI collaboration. The project is part of the Physics Beyond Collider (PBC) process presently carried out at CERN, and the European Strategy for Particle Physics Update. A possible host site for the prototype EDM storage ring is either COSY or CERN.

The scope of the project is to provide for protons at a kinetic energy of $\mathrm{T}=30 \mathrm{MeV}$ an all-electric machine operation with simultaneous clockwise (CW) and counter-clockwise (CCW) orbiting beams of the machine. The circumference of the machine is about $100 \mathrm{~m}$. At $\mathrm{T}=45 \mathrm{MeV}$ using vertical magnetic fields superimposed on the radial electric fields in the deflector elements, frozen-spin operation for protons shall be possible.

Items to be studied with the prototype ring include:

- Storage time investigations.

- $\mathrm{CW} / \mathrm{CCW}$ operation.

- Spin coherence time studies.

- Polarimeter studies.

- Studies of magnetic moment effects due to imperfect shielding and artificially induced magnetic fields.

- A direct measurement of the EDM of the protong.

- Tests of stochastic cooling.

\subsection{Precision EDM-ring}

The first measurement of the deuteron EDM and the design and realization of a prototype EDM ring represent tow fundamental milestone towards the design and realization of the final high-precision ring with a EDM sensitivity goal of $10^{-29} \mathrm{e} . \mathrm{cm}$ or even better. This will necessarily require the use of clockwise $(\mathrm{CW})$ and counter-clockwise $(\mathrm{CCW})$ beams to remedy systematic errors like: radial magnetic fields, non-radial electric fields, vertical quadrupole misalignments, rf-cavity misalignments and unwanted field components. As a matter of fact, the main systematic error coming from an unwanted spin precession due to the MDM in radial magnetic fields (which is indistinguishable from the EDM signal) can be controlled to a very high accuracy in the CW-CCW scheme, as the very same radial magnetic field causes forces in different directions for two opposite beams (Fig. 12).

Also in view of the possible construction of a dedicated EDM ring, COSY constitutes an important test facility of many EDM related key technologies. Besides polarimetry, beam position 


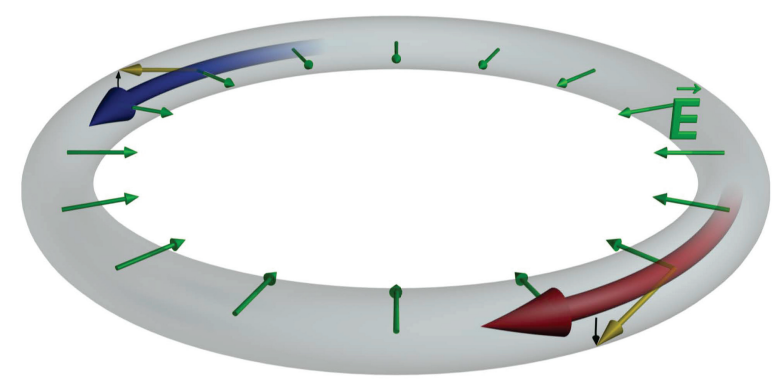

Figure 12: Concept of a dedicated ring for the measurement of an electrical dipole moment (proton case). The control of systematic effects suggests the use of two particle beams circulating in opposite directions in a pure electrostatic ring. Keeping the polarization vector aligned with momentum (frozen-spin condition), the interaction of an exisiting EDM with the electric field, would generate a torque slowly rotating the spin out of the plane of the storage ring into the vertical direction. Note that the EDM induced rotation will have different directions on the two beams. For the case of the proton, the frozen-spin condition is realized with a beam impulse of $\mathrm{p}=700.7 \mathrm{MeV} / \mathrm{c}$ (magic momentum). In this situation there is no spin precession in the accelerator plane due to the magnetic moment.

monitoring and active control systems, also the design of electrostatic and electromagnetic deflectors benefits by direct test in a storage rings. In addition, checks for systematic errors can be undertaken for further developments and applications.

\section{Acknowledgments}

The author wishes to acknowledge the support by the European Research Council via the ERC-AdG srEDM (Contract number 694340). He also thanks all involved members of the JEDI collaboration and of the Institut für Kernphysik of Forschungszentrum Jülich for their dedication and persistence towards this long-term project.

\section{References}

[1] see http://collaborations.fz-juelich.de/ikp/jedi/index.shtml

[2] JEDI collaboration, New method for a continuous determination of the spin tune in storage rings and implications for precision experiments, Phys. Rev. Lett. 115 (2015) 094801.

[3] JEDI collaboration, How to reach a thousand-second in-plane polarization lifetime with 0:97-GeV=c deuterons in a storage ring, Phys. Rev. Lett. 117 (2016) 054801.

[4] JEDI collaboration, Phase locking the spin precession in a storage ring, Phys. Rev. Lett. 119 (2017) 014801.

[5] JEDI collaboration, Spin tune mapping as a novel tool to probe the spin dynamics in storage rings, Phys. Rev. Accel. Beams 20 (2017) 072801.

[6] J. Slim et al., Electromagnetic Simulation and Design of a Novel Waveguide RF Wien Filter for Electric Dipole Moment Measurements of Protons and Deuterons, Nucl. Instrum. Meth. A828 (2016) 116 [1603.01567]. 
[7] V. Anastassopoulos, S. Andrianov, R. Baartman, S. Baessler, M. Bai, J. Benante et al., A storage ring experiment to detect a proton electric dipole moment, Review of Scientific Instruments 87 (2016) 115116.

[8] see http://sredm-ercgrant.de/ 